\title{
Molecular Beam Epitaxy-Grown InGaN Nanomushrooms and Nanowires for White Light Source Applications
}

\author{
A. Gasim ${ }^{1}$, T. K. $\mathrm{Ng}^{1}$, D. K. Cha ${ }^{2}$, P. Bhattacharya ${ }^{3}$, and B. S. Ooi ${ }^{1}$ * \\ ${ }^{I}$ Photonics Laboratory, Division of Physical Sciences and Engineering \\ ${ }^{2}$ Advanced Nanofabrication, Imaging and Characterization Core Laboratory \\ King Abdullah University of Science \& Technology (KAUST), Thuwal 23955-6900, Saudi Arabia \\ ${ }^{3}$ Department of Electrical Engineering and Computer Science, \\ University of Michigan, Ann Arbor, MI 48109-2122, USA \\ *E-mail: boon.ooi@kaust.edu.sa
}

\begin{abstract}
We report the observation of coexisting InGaN nanomushrooms and nanowires grown via MBE. Photoluminescence characterization shows that the nanostructures emit yellow and blue light, respectively. The combined emission is promising for white-LEDs.
\end{abstract}

\section{Introduction:}

The development of solid-state lighting is largely dependent on the fabrication of InGaN-based LEDs [1, 2]. However, the lack of a native substrate, which results in a high epitaxy defect density, has caused InGaN-based devices to suffer from efficiency droop at high drive currents. Recently, it has been demonstrated that InGaN nanowires (NWs) grown on a foreign substrate do not suffer from the lattice mismatch, allowing for the growth of high crystalline quality structures [3]. This ushered in intense research on InGaN NWs for solid-state lighting applications $[4,5]$. In this study, we report on the first observation of the coexistence of wurtzite InGaN NWs and nanomushrooms (NMs) grown on a silicon substrate using plasma-assisted molecular beam epitaxy (MBE). The NMs are comprised of a slender nanowire body and a wider flat hexagonal cap. To the best of our knowledge, this is the first observation of such nanostructures.

\section{Experimental Methods:}

The sample was grown using a radio-frequency N-plasma-assisted MBE system equipped with standard Ga and In sources with growth conditions similar to [6]. The InGaN nanostructures were grown on a (111) silicon substrate with a $150 \mathrm{~nm}$ buffer layer of $\mathrm{GaN}$. The optical and structural properties of the nanostructures were studied via micro-photoluminescence ( $\mu \mathrm{PL})$ and scanning electron microscopy (SEM).

\section{Results and Discussion:}

Fig.1 shows SEM images of the NMs. Fig.1(a) is a close-up image of a circular region that is predominated by NMs. Fig.1(b) provides a top-down view of the NMs, displaying the clear flat hexagonal shape of the cap-an indicator of high crystalline quality. The NMs appear to grow along the (001) direction, and the side facets of the cap represent the $\{100\}$ planes.
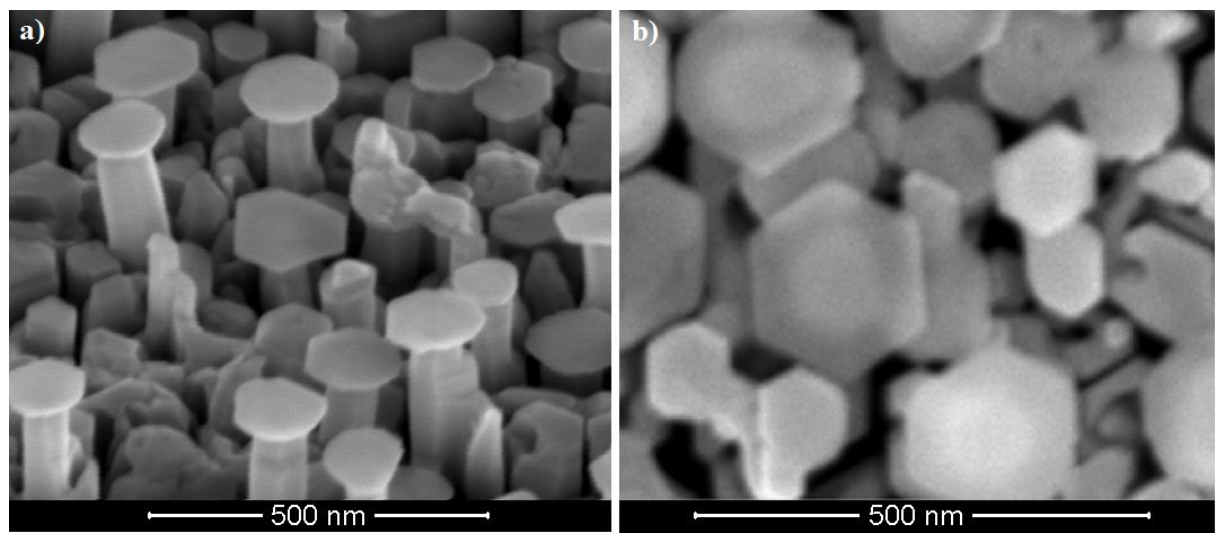

Fig. 1. (a) SEM image of the nanomushrooms; the large variation in size and height is evident (b) Top-down view of the nanomushrooms, displaying their flat hexagonal cap. The nanomushrooms grow in the (001) direction and the side facets represent the $\{100\}$ planes. 

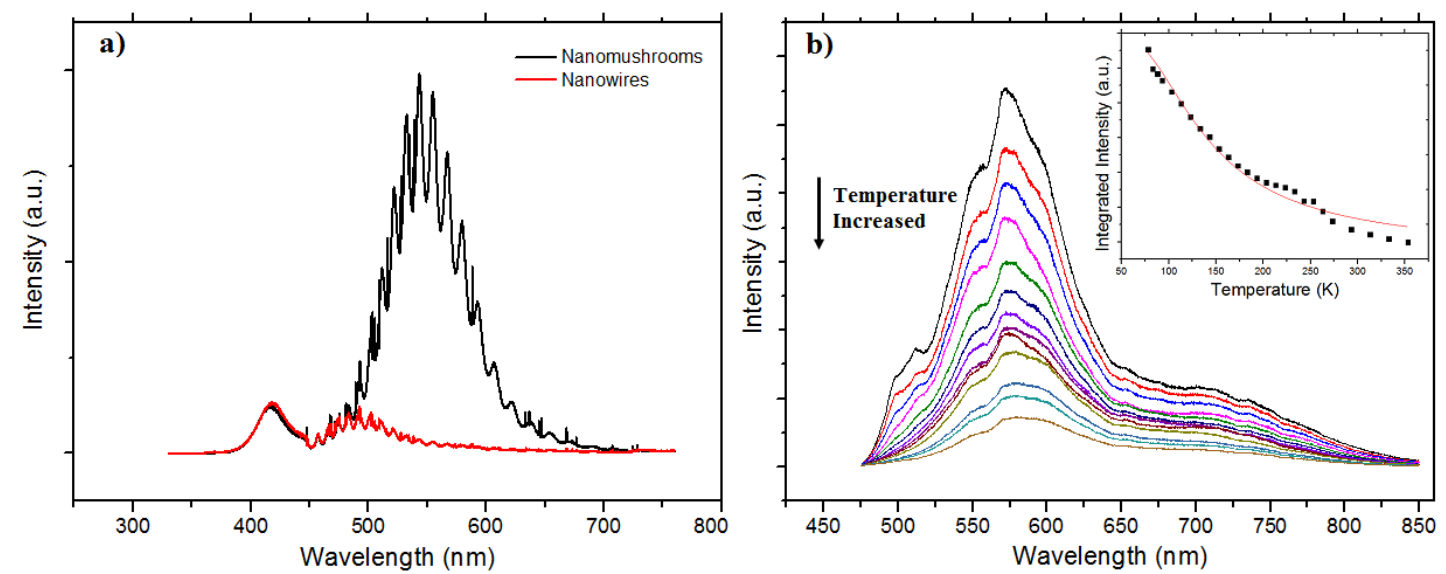

Fig 2. (a) A comparison between the spectra of the nanowires and nanomushrooms (excited using a 325nm laser). Both nanostructures lie on the same substrate; the ripples in the spectra are a consequence of the formation of standing waves in the substrate (b) Temperature-dependent PL spectra of the nanomushrooms (excited using a 473nm laser); the inset shows the integrated intensity plotted against the temperature.

The $\mu \mathrm{PL}$ characterizations for NMs and NWs are summarized above. Fig.2(a) shows both the significantly stronger emission and the large red shift in peak wavelength observed between the NWs and NMs. The redshift in wavelength might be attributed to higher InN molar fractions in the NMs. Recent studies have shown that the growth of $\mathrm{GaN}$ nanostructures is highly dependent on two factors: the temperature and surface diffusion kinetics [7, 8]. A hypothesis to explain the growth of the NMs is that a local modification to the substrate surface prior to growth may have influenced the local surface diffusion kinetics of the Ga and In adatoms, resulting in nanostructures with different shapes and compositions. Studies to determine the exact cause of the growth of the NMs remain on-going.

Fig. 2(b) shows the temperature-dependent PL spectra for the NMs and the corresponding integrated intensities plotted against the temperature. The theoretical fit in the inset was used to extract the activation energy of the nonradiative centers, which was found to have a value of $32 \mathrm{meV}$. This large value, which agrees with the NW value, is a corollary of large In fluctuations, which engender potential minima that promote radiative recombination. Of greater interest is the extracted internal quantum efficiency (IQE) of the NMs, which was roughly $17 \%$. This value was significantly greater than that of the NWs grown on the same sample $(\sim 10 \%)$, indicating that the NMs are more efficient structures, although it should be noted that the IQEs of some InGaN NWs in the literature have reached 20$30 \%$ [4]. Ultimately, the growth of NMs alongside NWs in a single-layer growth instead of conventional compositional variation in multi-layer growth could potentially pave the way to high-efficiency white LEDs.

\section{Summary:}

We report the observation of coexisting InGaN nanomushrooms and nanowires grown via MBE. Photoluminescence characterization shows that the nanostructures emit yellow and blue light, respectively. The combined emission is promising for white-LEDs.

\section{References:}

[1] T. Mukai, M. Yamada, and S. Nakamura, "Characteristics of InGaN-based UV/blue/green/amber/red light-emitting diodes," Japanese Journal of Applied Physics, vol. 38, pp. 3976-3981, 1999.

[2] E. F. Schubert and J. K. Kim, "Solid-state light sources getting smart," Science, vol. 308, pp. 1274-1278, May 2005.

[3] L. Cerutti, "Wurtzite GaN nanocolumns grown on Si(001) by molecular beam epitaxy," Applied Physics Letters, vol. 88, pp. 213114-3, 2006.

[4] W. Guo, M. Zhang, A. Banerjee, and P. Bhattacharya, "Catalyst-free InGaN/GaN nanowire light emitting diodes grown on (001) silicon by molecular beam epitaxy," Nano Letters, vol. 10, pp. 3355-3359, Sep 2010.

[5] R. Armitage and K. Tsubaki, "Multicolour luminescence from InGaN quantum wells grown over GaN nanowire arrays by molecularbeam epitaxy," Nanotechnology, vol. 21, 2010.

[6] M. Zhang, P. Bhattacharya, and W. Guo, "InGaN/GaN self-organized quantum dot green light emitting diodes with reduced efficiency droop," Applied Physics Letters, vol. 97, pp. 011103-3, 2010.

[7] K. A. Bertness, A. Roshko, N. A. Sanford, J. M. Barker, and A. V. Davydov, "Spontaneously grown GaN and AlGaN nanowires," Journal of Crystal Growth, vol. 287, pp. 522-527, 2006.

[8] R. Mata, K. Hestroffer, J. Budagosky, A. Cros, C. Bougerol, H. Renevier, and B. Daudin, "Nucleation of GaN nanowires grown by plasma-assisted molecular beam epitaxy: The effect of temperature," Journal of Crystal Growth, vol. 334, pp. 177-180, 2011. 